\title{
Neige ardente ou les métamorphoses des éléments dans les littératures maghrébines d'expression française
}

\section{Labra-Cenitagoya, Ana Isabel}

Universidad de Alcalá, anai.labra@uah.es

\begin{abstract}
Resumen
La literatura magrebí, en general, y argelina, en particular, mantienen una estrecha relación con la naturaleza y los elementos (tierra, agua, fuego, aire). Sería posible ver en esta tendencia la huella de una visión ancestral del mundo, de una aproximación a lo real que podríamos calificar de mística en un sentido amplio del término. Entre los diferentes elementos que componen cuanto existe, hay que señalar en el caso de estas literaturas la omnipresencia de los elementos fuego y agua declinados en todas sus variantes y combinados de las formas más sorprendentes. La presencia constante del sol en los paisajes magrebíes y la escasez, que de ella se deriva, del agua imprescindible para la vida condicionan desde hace siglos el imaginario artístico en estas latitudes. Los escritos y escritores no escapan a esta dinámica creadora. En efecto, en las producciones literarias magrebíes abundan las imágenes elaboradas a partir de estos dos elementos, oponiéndolos o fusionándolos para dar lugar a metamorfosis que ofrecen a sus lectores una visión diferente de la realidad, otra lectura del mundo y de los seres. Este artículo ofrece una aproximación a esta tendencia de la poética magrebí antes de pasar al análisis detallado de algunos ejemplos tomados de las novelas de los escritores argelinos Mohamed Dib y Assia Djebar.
\end{abstract}

Palabras clave : literaturas magrebíes de expresión francesa ; elementos naturales ; metamorfosis ; Dib ; Djebar.

\begin{abstract}
Résumé
La littérature maghrébine, en général, et algérienne, en particulier, entretiennent un rapport étroit avec la nature et les éléments (terre, eau, feu, air). Il serait possible d'y déceler des traces d'une vision ancienne du monde, d'une approche du réel que nous pourrions qualifier de mystique dans le sens large du terme. Parmi les différents éléments qui composent l'existant, il faudrait souligner dans le cas de ces littératures l'omniprésence des éléments feu et eau déclinés dans toutes leurs variantes et combinés des façons les plus surprenantes. La présence constante du soleil dans les paysages maghrébins et la rareté conséquente de l'eau nécessaire à la vie conditionnent depuis des siècles l'imaginaire artistique de ces contrées. Les écrits et les écrivains n'échappent pas à cette dynamique créatrice. En effet, les productions littéraires maghrébines foisonnent d'images élaborées à partir de ces deux éléments, les opposant ou les fusionnant pour donner lieu à des métamorphoses qui offrent aux lecteurs une vision différente de la réalité, une lecture autre du monde et des êtres. Cet article offre un panorama de cette tendance de la poétique maghrébine avant de passer à l'analyse détaillée de quelques exemples extraits des romans des écrivains algériens Mohamed Dib et Assia Djebar.
\end{abstract}

Mots-clés : littératures maghrébines d'expression française ; éléments naturels ; métamorphose ; Dib ; Djebar.

\footnotetext{
Abstract

Maghrebian literature in general and Algeria in particular have a close relationship with nature and natural elements (earth, water, fire, air). A reading of this trend could make possible for readers to identify a mark of an ancient worldview and of a mystical way to approach the real, in a broad sense of the term. Among the different elements that shape the world, the pervasiveness of fire and water in its different variations and most unexpected combinations is particularly meaningful in Maghrebian literature. The constant presence of the sun in the Maghreb landscapes and its derived absence of nurturing water seem to have shaped the artistic imagination for centuries in these latitudes. Writings
} 
and writers do not escape these creative dynamics. Indeed, images inspired in the two elements abound in Maghrebian literary productions, either opposing or merging fire and water to trigger a metamorphosis that offers readers a different view of reality, a new reading of the world and its peoples. This article focuses on this Maghrebian poetic trend before thoroughly analysing a selection of examples from novels by Algerian writers, Assia Djebar and Mohamed Dib.

Keywords : maghrebian francophone literatures ; natural elements ; metamorphose ; Dib ;Djebar.

Je me battrai avec du sable et de l'eau. De l'eau fraîche, du sable chaud.

(Kateb Yacine, Nedjma, 1956 : 53-54)

\section{Introduction}

Dans la communication intitulée « Lumière sur lumière » que je présentais en 2013 au XXII Colloque de l'APFUE sur les fonctions de la métaphore lumineuse dans la narrative des écrivains algériens Mohamed Dib et Assia Djebar j'avançais que :

Les motifs eau et lumière privilégiés par ces deux auteurs pendant la première période sont toujours présents dans les romans de cette deuxième période mais, nous allons le voir, Dib et Djebar vont finir par les fondre en un seul au moyen d'images rassemblant, par exemple, le ciel et la mer, l'homme et la femme, les animaux-les plantes-les choses et l'être humain. C'est ainsi que, dans les analogies dont se servent ces deux auteurs, le principe mystique de la fusion/dissolution dans l'Absolu, dernière étape d'une vision mystique du réel, semble s'imposer progressivement (sous presse).

Mais cette analyse se consacrant aux métaphores basées sur le sème lumière, j'ai dû alors passer sur la présence fondamentale et constante de l'élément aquatique dans ces deux auteurs et ses rapports avec d'autres éléments naturels comme la terre et l'air.

Pour comprendre l'utilisation que les auteurs maghrébins en général et ces deux auteurs en particulier font de la nature, il faut rappeler que l'espace est une composante définitoire des littératures postcoloniales Selon Jean-Marc Moura, dans son essai Littératures francophones et théorie postcoloniale :

[L]'œuvre francophone construit d'une manière insistante son espace d'énonciation: c'est l'un des signes les plus manifestes des littératures coloniales et postcoloniales. L'œuvre qui choisit de gommer ses relations à cet espace vise en effet rien moins qu'une inscription dans la tradition européenne, en effaçant toute trace linguistique, thématique, formelle de son origine (1999: 129).

Le rapport à l'espace serait ainsi l'une des déclinaisons de la thématique postcoloniale par excellence : l’identité personnelle et collective. Au point que l'écrivain algérien Mohamed Dib se demande « Suis-je mon paysage ?ı

\section{L’écriture maghrébine entre le désert et la mer}

Les littératures maghrébines naissent dans le cadre méditerranéen, caractérisé par des paysages qui vont des zones côtières où la mer impose une végétation et un climat doux au cœur du désert chaud le plus grand au monde, le Sahara (dont le nom signifie désert en arabe), tout en passant par la chaîne montagneuse de l'Atlas avec des sommets enneigés qui dépassent les 4000 mètres. Le Maghreb se situe, sur les cartes mais aussi dans les imaginaires, entre le désert et la mer, tel que le présente l'historien amoureux de l'Algérie Emilio Sola, dans un intéressant essai éponyme. De l'eau à la neige pour finir dans le sable, toujours sous le soleil africain siégeant implacable dans un ciel d'un bleu méditerranéen. 
Une évolution des éléments se dessine déjà dans cette simple approche du paysage maghrébin. Et c'est ce même trajet imaginaire que je m’apprête à réaliser ici guidée par quelques auteurs maghrébins qui essaient de dessiner dans leurs ouvrages une vision du réel et de l’identité personnelle dans toute leur complexité.

Il y a quelques années, l’écrivain algérien Amin Zaoui affirmait dans un article publié par le journal Liberté Algérie :

La littérature maghrébine et arabe, écrite dans les trois langues : l'arabe, le tamazigh et le français, est entourée de milliers de kilomètres d'eau. Des montagnes de vagues ! De partout il y a que des mers et des océans. Mais cette eau ne parle pas aux écrivains. [...] Nos textes littéraires sont à sec. Ils sont en bois ou presque ! Sablés ! Nous écrivons comme sur le dos d'un chameau. Selon les rythmes des pas des chameaux, sont nés nos poèmes ! Nous traversons encore l'ère du sable. Beaucoup de sable dans les pages de nos livres. Nos poètes ont le regard braqué sur le Sud. La mer est un espace d'échange, d'ouverture et d'attente. Le désert lui aussi a son sens d'évasion et d'aventure. Combien de romanciers algériens, maghrébins ou arabes ont-ils les pieds dans l'eau de la mer ? Ont-ils des ailes aquatiques ? (2008).

Et d'insister encore : «Le rapport à la mer, exprimé dans nos quelques textes littéraires ou picturaux, demeurera marginal ou superficiel. Dès que l’image de la mer est présente dans la littérature maghrébine d'aujourd’hui, elle est directement liée au phénomène d'“el-harga”. Une autre forme de suicide. La mer est vidée de sa beauté. » (Zaoui, 2008)

Il paraîtrait donc que le désert l’importe finalement sur la mer. Comme le dit si bien Mohamed Dib dans son essairoman Simorgh : «Le désert qui se dresse à notre horizon géophysique se dresse à notre horizon mental » (2003a : 91).

Mais le jugement de Zaoui est peut-être un peu hâtif ou trop absolu car il est aisé de trouver dans les littératures du Maghreb d'autres auteurs donnant à l'eau, à la mer, une place privilégiée dans leurs écrits. Et toujours, chez eux, ce vaet-vient entre la mer et le désert, entre l'eau et le sable.

Selon Ana Monleón dans son article « L’interculturalité dans l’univers poétique de Tahar Bekri », l'eau, la lumière et la terre sont trois axes symboliques majeurs qui traversent toute l’œuvre du tunisien Tahar Bekri. La mer Méditerranée est, sans aucun doute, le centre qui structure cette architecture imaginaire où « le poème est une mer qui prend feu » (2007 : 185) et un attribut indissociable de l'identité de l'auteur. C’est ainsi par exemple que son recueil La brûlante rumeur de la mer se structure autour de ce thème de la mer.

Dans le cas de l’algérienne Malika Mokaddem, le mouvement est l’inverse. $N$ 'zid, son sixième roman, a été publié en 2001 aux Editions du Seuil. Il relate l'histoire d'une jeune femme, Nora Carson, qui se trouve en pleine mer Méditerranée sans mémoire, sans passé. Et c’est à travers des rencontres au milieu de cette étendue maritime quelle pourra constituer/reconstituer sa véritable identité. Après avoir centré toutes ses intrigues précédentes autour d’un désert d’énigmes, ce n'est qu'avec $N^{\prime}$ zid que Malika Mokeddem change de décor, situant la mer ${ }^{1}$ au centre du roman.

Ce recours obsédant à ces deux espaces apparemment contradictoires, le désert et la mer, pour métaphoriser l'identité autant personnelle que collective est également de mise dans les romans des deux grands noms de la littérature algérienne, Mohamed Dib et Assia Djebar. Les exemples sont trop nombreux et nous n’avons pas ici la possibilité de les évoquer tous, mais nous pourrions par exemple évoquer l'utilisation du sable et de l'eau (avec sa variante la neige) pour se référer à la mémoire, la parole et l'écriture, ces outils dont se servent les écrivains postcoloniaux pour lutter contre l’oubli et l'acculturation dérivés de l'expérience coloniale:

Revient en nous le lent, l'obsédant bruit de l'eau, à vous de savoir s'il s’agit de l'antienne effrénée de la mer, peuples des rives méditerranéens, ou seulement de l’eau tassée, assourdie, des maigres rigoles, des puits à demi desséchés

\footnotetext{
${ }^{1}$ Cette transition est analysée par le professeur Zoubida Belaghoueg dans son article « Malika Mokeddem. Du sable et de l’eau : jaillissement de la création et itinéraire d'une vie ». L'importance de cette dimension spatiale ainsi que l'identification des deux espaces (désert-mer) résultent evidentes dans ce roman de Mokkedem : « Elle naviguait sur une mer déchainée. Des lames croisées menaçaient de disloquer le bateau. De gros nuages crevaient le ciel. Les vents tournants de l'orage soufflaient, soulevant de monstrueuses tornades d'eau. Peu à peu, toute cette fureur liquide a commencé à devenir rouge, un rouge sang. La tempête s'est muée en vent de sable. La mer s'est coagulée en désert » (Mokeddem, $2001: 65$ ).
} 
[...] des oueds égarés dans les franges désertiques, déplacement inéluctable non du passé au présent, plutôt du nord au sud [...] Mémoire de l'eau, plutôt mémoire des sables, silence... (Djebar, 1999 : 158).

\section{Les valeurs de l'eau dans l'imaginaire maghrébin}

L'eau est un symbole avec une nature ambivalente, en même temps positive et négative, profane et sacrée. Comme le rappelle Carine Bourget dans son essai Coran et tradition islamique dans la littérature maghrébine reprenant les mots de Chebel dans son Dictionnaire des symboles musulmans, «l'eau est un élément à la symbolique double. [...] L’eau dans le Coran est source de vie, mais dans l'imaginaire populaire certains lacs et eaux stagnantes sont maléfiques » (1995 : 115). Dans leurs romans écrits pendant et sur la période coloniale en Algérie, Dib et Djebar vont jouer sur cette double nature bénéfique et destructrice de l’élément aquatique.

Ainsi le peuple aux armes est souvent représenté par Dib comme un torrent d'eau (parfois de sang, un autre élément liquide) ou comme un raz-de-marée : « Autour d'elle, on ne savait quoi grondait dans le cœur des montagnes et des vallées [...] l'on entendait sonner jusqu'au fond de l'horizon ce torrent de forces captives qui allait un jour inonder le pays » (2002: 175-176).

Djebar, de par sa condition personnelle en tant que femme et bourgeoise, va vivre la guerre en exilée dès la frontière tunisienne comme une longue attente. Elle va donc privilégier l'image de l'eau étanche, d'un marécage d'eau pourri et immobile : «Cette puanteur après l'avoir maudite des jours entiers [...] il m'arrive quelquefois de la comparer à la présence de la guerre. Car la guerre pourrit en nous. » (1983 : 195).

Une image qui sera reprise par Dib quelques années plus tard pour caractériser l'Algérie post révolution, l’Algérie de la révolution trahie : « Nos villes, toutes nos villes, ne sont que marais, eaux mortes sur lesquelles les démons de la foi et la soumission veillent » $(1978: 90)$.

Une autre métaphore de l'eau partagée par ces deux auteurs est celle de l'Algérie de preguerre comme un bateau sur le point de partir :

Les contours du pays s'enfoncèrent à l'arrière-plan des brouillards d'été. Les champs voguaient, ayant rompu leurs amarres. Agrippé à sa quille d'argile, le village des Bni Boublen inférieur prenait le départ, cinglait en plein ciel (Dib, $2002: 15)$.

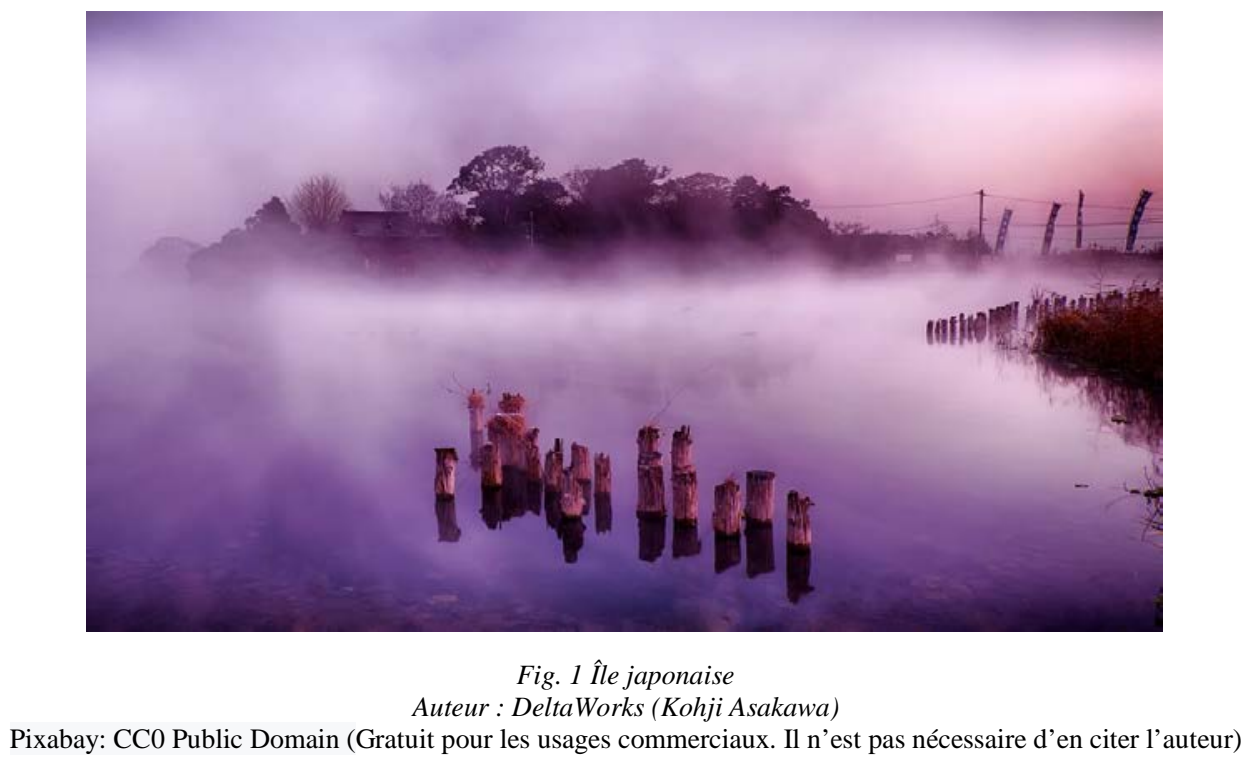

La vieille ville, à force de voir cette campagne ressembler à quelque immense coque de navire noyée dans la cruauté de l'histoire, s'énorgueillit d’être seule à suivre les racines qui la relient aux générations passées; mais ainsi fixés au 
milieu de la dérive, les gens de ces familles ne s’aperçoivent pas que peut-être épargnés du naufrage, ils ont dû se fermer sur eux-mêmes (Djebar, 1962: 205).

Mais l'image liquide privilégiée par ces deux auteurs est celle de la mer, qui prend selon les romans et les époques des fonctions et des formes différentes, aussi changeante que la réalité naturelle qui lui sert de base. Dans leurs romans sur l'époque coloniale et la guerre d'indépendance, le peuple sera identifié à la mer de par sa force écrasante et son aspect compact et infini. Une métaphore à caractère universel que nous pouvons retrouver aussi par exemple dans les écrits de la philosophe espagnole María Zambrano: "Así es la Patria, Mar que recoge el río de la muchedumbre. Esa muchedumbre en la que uno va sin marcharse, sin perderse, el Pueblo» (1989 : 91).

Une autre signification de l'eau qui fait partie de l'imaginaire collectif est celle du temps qui passe ou qui s'arrête, celle de la Vie humaine. Cette correspondance exacte se produit au niveau superficiel de la durée biologique comprise comme un parcours avec un début et une fin : «nuestras vidas son los ríos... » Mais cela est vrai aussi à un niveau plus profond, celui des grandes questions auxquelles toutes les religions essaient de répondre. Dans notre culture judéochrétienne par exemple, le Christ dit de lui-même «je suis une source d'eau vive et qui boira de cette eau n’aura plus jamais soif ». Revenant dans un contexte culturel musulman, le maître soufi Ibn Arabi considère l'eau comme le symbole le plus exact de la vie car elle est présente dans toutes les réalités. Elle correspond ainsi à l'Ipséitéde l'Absolu (Izutsu, 1997 : 171).

D’un autre côté, «[1]'eau, élément essentiel pour sustenter la vie matérielle [et cela est spécialement vrai dans les contrées désertiques où la foi musulmane a vu le jour], symbolise dans l'Islam [...] la foi et la vie spirituelle.» (Bourget, 2002 : 120). «La révélation divine est comparée à de l'eau à maintes reprises dans le Coran. Cette valeur intrinsèque et transcendantale de l'eau est exemplifiée dans le rituel de purification qui doit précéder la prière. » (Bourget, $2002:$ 120)

Voyons un exemple littéraire : l'écrivain d'origine marocaine Abdelkader Benali construit son premier roman Noces à la mer autour de cette valeur symbolique de l'eau comme élément purificateur mais aussi de vie et de fertilité. Le jeune Lamarat vit en Hollande mais il revient au Maroc pour assister au mariage de sa sœur Rébecca avec leur oncle Mosa. Mais, avant le mariage, Mosa disparaît. Il sera retrouvé par les deux frères dans les champs, endormi et ivre après plusieurs jours de débauche dans le prostibule de la région. Afin de le punir mais aussi de s’assurer une future fidélité, la mariée coupe le gros bout du sexe de Mosa sur le sable de la plage et puis entre avec lui dans l'eau pour célébrer leurs noces à la mer, un étrange rituel de purification :

“Et maintenant, cher homme et époux, je t'emmène dans l'eau, corps et âme, pour laver ton ivrognerie et le sang du sacrifice.” Ils étaient debout dans l'eau peu profonde. Rébecca se retourna et tira à elle Mosa, qui était en train de perdre, avec son sang, son honneur, sa force et tout ce qui fait d'un homme un homme,

aux noces à la mer, aux noces à la mer.

Ils ne sortirent de l'eau que lorsqu'elle estima que la nuit de noces était consommée (1996 : 244).

Malek Chebel explique dans son Dictionnaire des symboles musulmans que «[d]ans la terminologie sexuelle, l'eau est une métaphore du sperme, appelé al-ma [de l'eau en arabe] [...] L'eau [...] participe à un grand nombre de pratiques incantatoires [elle] est également de rigueur dans toutes les prophylaxies maternelles, notamment en ce qui concerne la protection du jeune nourrisson » (2002: 149).

Dans le Coran, beaucoup d'éléments naturels sont des signes, des préuves de la miséricorde divine, mais surtout l'eau et la lumière. "Être à l'écoute de la terre et de la nature, c'est être à l'écoute de Dieu dont elle est signe tout comme les versets du Coran" (Chebel, 2002 : 157). Dans son roman La Mère du printemps, Chraïbi se sert de la technique rhétorique arabe appelée iqtibas qui consiste à reproduire de façon littérale ou approximative des phrases su Coran. Le narrateur Azwaw-Filani clôt le roman avec une réflexion où Dieu et la terre natale se superposent et se confondent dans leur nature éternelle face à la limitation de la vie humaine: «"Quand il ne subsistera plus rien, il subsistera la Face 
Sublime de Dieu.” C’est ce qu'affirme le Coran, qui chante dans mon cœur. Les peuples passeront comme une caravane le long du temps. Et au bout du temps, il y aura toujours la terre, la lumière et l'eau de mon pays » (1982 : 214$).$

Mohamed Dib se sert aussi de l'iqtibas dans Le Sommeil d'Ève mais, dans son texte, il confond apparemment les termes lumière et eau. En réalité, cette confusion cherchée lui permet d’incorporer le paysage finlandais (où se situe l’histoire et où l'auteur a passé de longues périodes) à son identité culturelle méditerranéenne et musulmane.

Un pays qui va de lac en lac, ne s’accommode, semble-t-il, que de cette eau dormante [...] "N'as-tu pas regardé comme le Seigneur étend sa lumière ? S’il le voulait, elle resterait à demeure. Nous avons commis l'eau pour preuve.” Non, mais que suis-je en train de débiter là ? L'authentique, la vraie parole est : N'as-tu pas regardé comme ton Dieu étend l'ombre ? S’il le voulait, elle resterait à demeure. Nous avons commis le soleil pour preuve... (Dib, 2003b : 195).

Cette double nature de l'eau (à l'origine de la vie matérielle mais aussi pont qui relie la réalité et le sacré) la rend susceptible d'offrir à l'homme des réponses, même incompréhensibles, aux questions sur l'existence. Dans les mots de la méditerranéenne María Zambrano « [...] nada hay como el elemento acuoso para desatar esa atención, esa ansia de escuchar y esta esperanza informulada de que de las aguas [...] llegue a surgir algo » (Zambrano, 1977 : 145-146). Et en écho, un méditerranéen de l’autre rive, Mohammed Dib, semble lui répondre :

Quelqu'un compte-t-il ses jours : cela ne nuit en rien à la révélation. Se le dire, s’entretenir dans le dialogue de l'âme avec l'eau, s'immerger dans sa fraîcheur. Opaque, le monde a choisi sa transparence pour se dévoiler à nous qui en restons l'ombre-l'ombre qu'il projette et qui le trouble [...] l'eau répond et revient à la charge avec des nouvelles réponses (1995: 147).

\section{L’écriture éphémère de la nature et la métamorphose des éléments}

Le monde semble rester muet face au désarroi des êtres humains. Mais en réalité c’est le monde tout entier qui répond, bien que de façon énigmatique, à ces questions posées par les hommes. La seule réponse possible semble être l'écriture éphémère de la nature, les signes tracés dans les nuages, la neige ou le sable. Ces réponses offertes par les éléments naturels sont appelés atlals par la tradition arabe: "Ce sont les nuages avançant là-haut en rangs serrés qui entretiennent une inquiétude sans visage. Ils semblent eux aussi partir à la recherche d'un objet perdu, sans doute d'un être perdu. Ce ciel est une écriture qui en recouvre une autre. Cela pour le traducteur que je suis » (Dib, 1990: 61). « Retourne là où tu as déposé la bête et lis ce qu'elle a écrit. Des atlals à n’en pas douter. Va fillette. Elle n’y est plus. Le sable est redevenu la page blanche, nette, qu’il a été et sera à jamais. Le vent, ce vent qui ne reprend haleine à aucun moment, l’a soufflé » (Dib, 1994: 159).

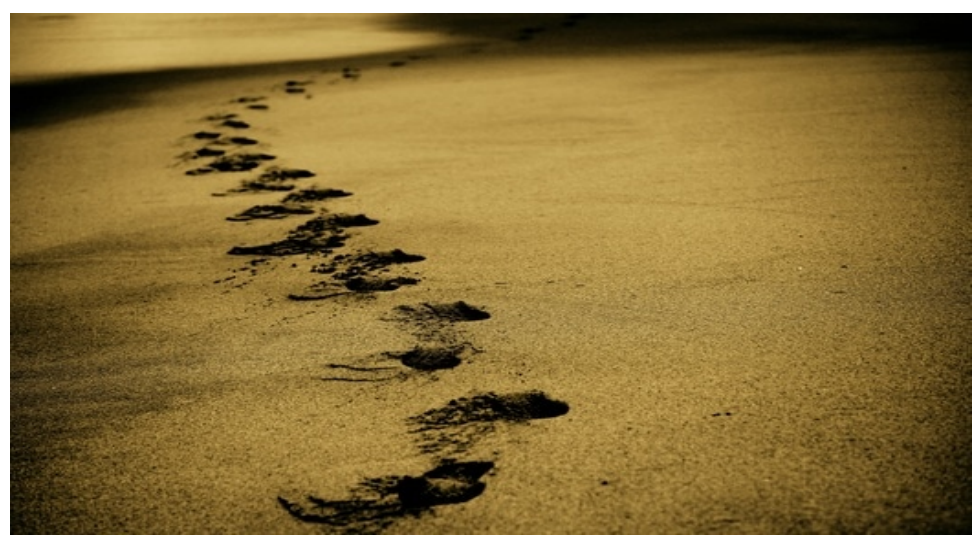

Fig.2 Traces sur le sable

Pexels : CC0 License (Gratuit pour les usages commerciaux. Il n’est pas nécessaire d’en citer l’auteur) 
Dans cette réponse, les éléments naturels se mélangent, se métamorphosent le uns dans les autres rendant évidente l'unicité du réel, doctrine de base de tous les mysticismes : « Le soufisme et l'art musulman connaissent cette tablité, ou tout s'unit et répond à tout : [...] car la doctrine de l'unité (at-tawhid) est le fondement même de l’Islam » (Arabi, 1989: 263). Dans les lignes qui suivent, je voudrais m’attarder sur quelques exemples extraits des romans de Mohamed Dib et d’Assia Djebar se référant à cette métamorphose des éléments naturels.

J’ai déjà signalé que les constellations métaphoriques de l'eau et du sable servent à Dib et à Djebar pour se référer à l'écriture et à la mémoire. Mais elles servent aussi à exprimer leurs contraires : le silence, l'oubli ou le vide. Ainsi Djebar, dans son roman Les Nuits de Strasbourg, décrit la ville de Strasbourg couverte par la neige comme « un vide... horizontal, un creux sonore impressionnant» (1997 : 395). Et dans L’Amour la fantasia, le silence d'une jeune femme sur son viol devient « du sable brûlant éparpillé sur tout parole » (1995: 226). Mais le lecteur va retrouver des images où la neige se métamorphose dans d’autres éléments ajoutant des significations a cette valeur initiale :

La neige cendre : « Le silence retomba et, comme une cendre froide, ensevelit tout dans la pièce » (Dib, 1995 : 189).

La neige terre : « $[\ldots]$ tous deux nous finiront sous une neige noire qui nous couvrira avant de tout rendre à l'innocence blanche » (Dib, 1995 : 100).

Et surtout, la neige sable : «Certaines façons d'être de la lumière recouvrent parfois le désert d'une blancheur de neige » (Dib, 1994 : 156). « Notre sable à nous, c’est la neige. Ainsi, je connais la neige et le sable. Et quelque part ils sont frère et sœur. Pour l'instant, je suis au milieu de cette neige de sable toute chaude » (Dib, 1994: 147). « Thelja, mon chéri, signifie Neige ! [...] Ô neige, soupire-t-il, femme ardente qui me brûle ! (Djebar, 1997 : 58-59).

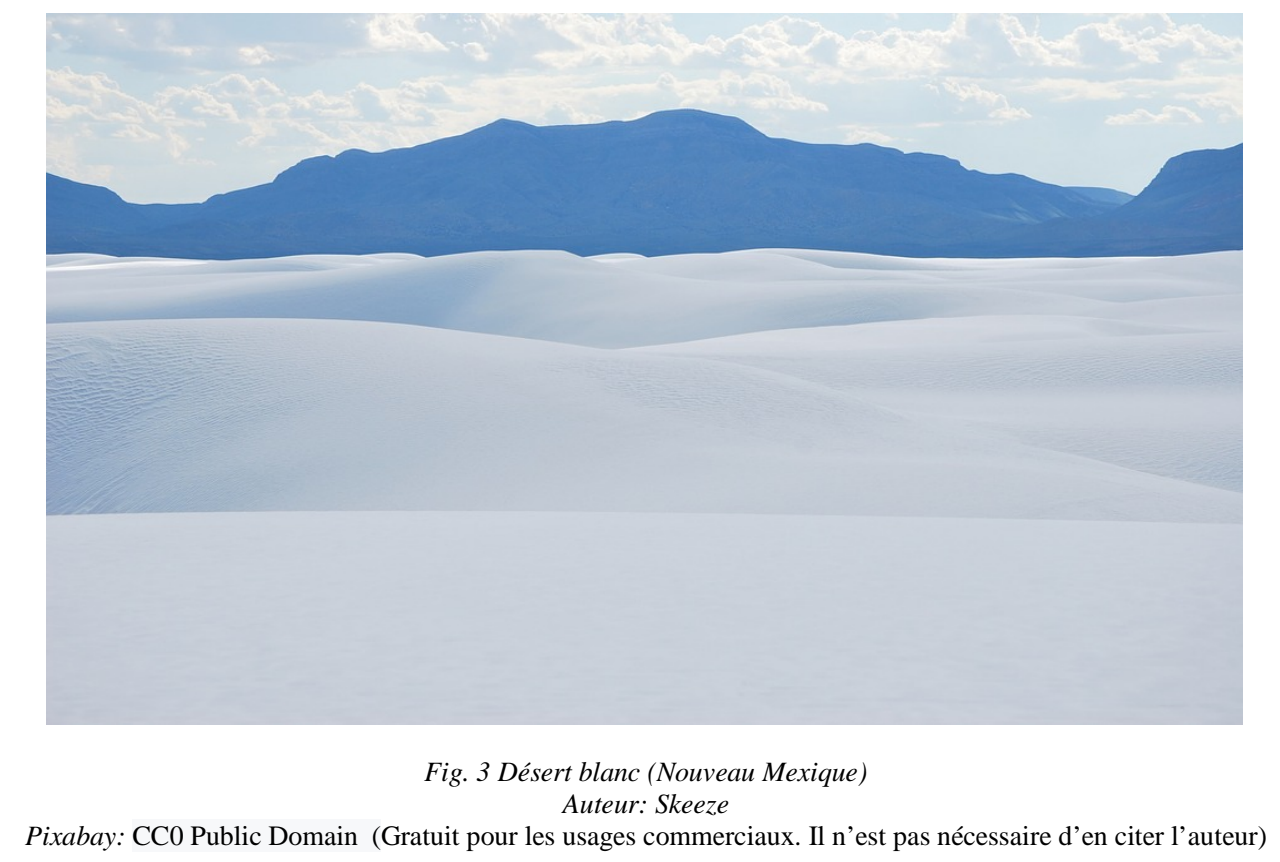

Mais, sans aucun doute, la métamorphose la plus porteuse de sens d'un point de vue littéraire et identitaire est celle de l'eau en lumière, ces deux signes privilégiés par le Coran. Eau et lumière confondues au service de la recherche de la vérité profonde de l’homme. Analysons en détail deux exemples.

La première métamorphose part d’un élément écrit : la lettre ‘ayn. Ce terme (mais ce n’est pas le seul cas, loin de là, dans la langue arabe, extrêmement riche en interprétations à ce niveau) est très polysémique. Chebel (2002 : 119) rappelle que, selon les contextes, ce mot peut se référer à l'organe de la vision (l'œil), à la dix-huitième lettre de l'alphabet arabe ou à une source d’eau. Ou à plusieurs de ces éléments à la fois. Bárcena signale que « [e]n árabe, la palabra ayn quiere decir ojo, fuente, origen, esencia, sí mismo. Ayn es el órgano de la visión interna directa e inmediata. También designa el disco solar (ayn ash-shams) » (2008). 


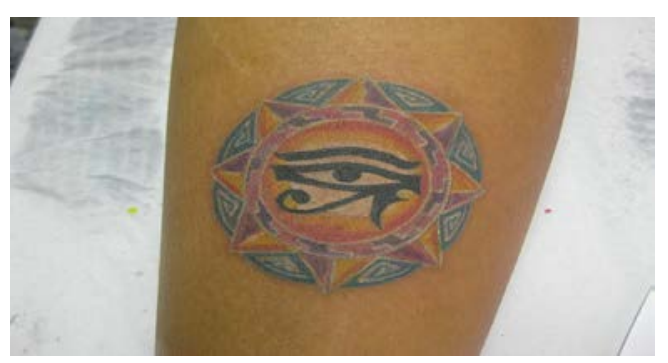

Fig. 4 CEil solaire (L'œil d'Horus). Talisman

photo credit: <a href="http://www.flickr.com/photos/12019015@N06/3927305112" > Tatuagem Horus eye tattoo $</ a>v i a<a$ href="http://photopin.com" $>$ photopin $</ a><a$ href="https://creativecommons.org/licenses/by/2.0/" $>($ license) $</ a>$

Dib et Djebar, écrivains de langue française mais de substrat culturel musulman, ont recours à plusieurs reprises et avec des modulations différentes à cette polysémie du terme ayn-œil. Tantôt l'œil sera eau : « [...] les yeux qu'elle ouvrait en grand : une eau et, au fond, le secret de l'eau » (Dib, 2003b : 186). «Un regard en crue. Et son eau noire pourtant claire, grave, comme si elle allait submerger l'espace autour » (Djebar, 2002 : 318). Tantôt l'eau deviendra œil : « Considérez ce ciel, puis considérez cette eau unie : vous n'en vibrerez pas moins. Calme dans son mutisme, le lac vous considère aussi, œil omniscient ouvert par-delà toute chose [...] Reconnaissez-la, elle vous reconnaîtra. Ma vie me paraît pleine de lumière, me paraît presque rachetée » (Dib, 1995 : 147). Tantôt le soleil sera un œil bienveillant ou terrible qui, du ciel, contrôle le monde : « Ce matin, un matin d'avril, déploie pourtant sur le monde le regard neuf d'un œil azuré tout de douceur humide, de fraîcheur, la voie semble ouverte, qui mène à l'été » (Dib, 2001 : 34).

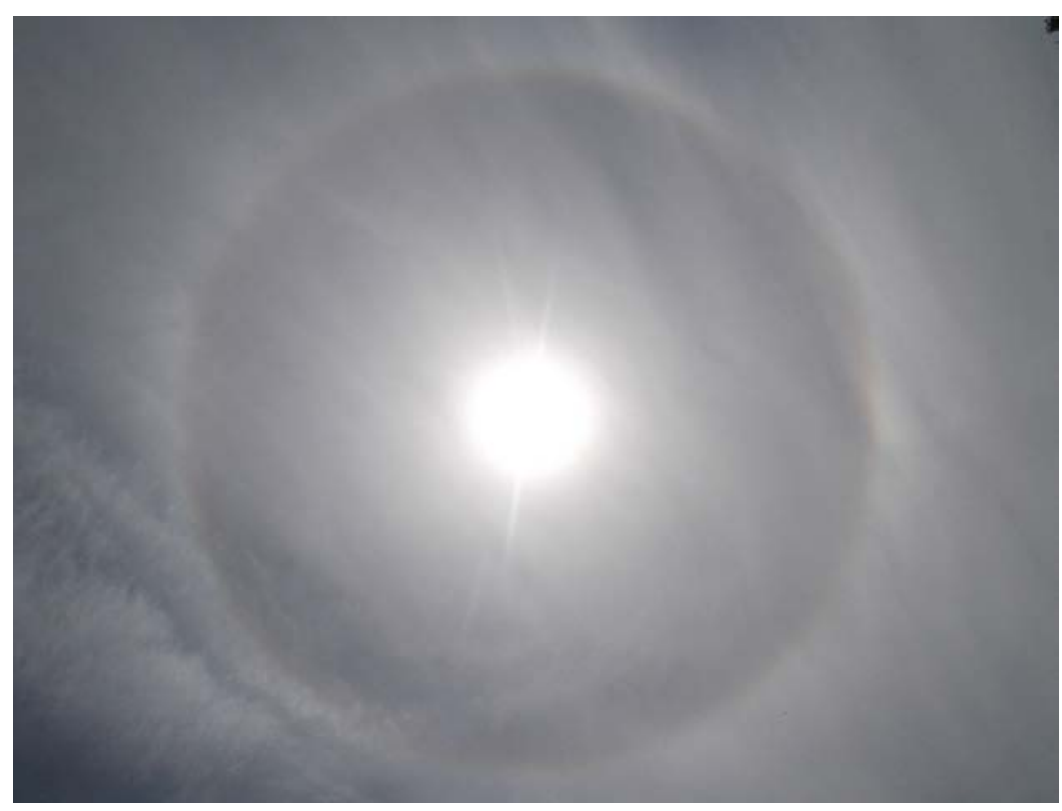

Fig. 5 Halo solaire

Auteur : cbo1964 (Carsten Bornhöft)

Pixabay: CC0 Public Domain (Gratuit pour les usages commerciaux. Il n’est pas nécessaire d'en citer l'auteur)

Moi, qui suis-je ? Mais, le soleil au zénith dont nul ne soutient la vue ! Le Chasseur inconnu enclos dans l'enceinte de la parole ! L’œil qui assèche l'espace. Le sable du présent avant le sable du passé. Le futur passé et présent. La splendeur. [...] Et le silence se reforme, plus lourd d’avoir été secoué par cette voix pléthorique, météorique, métaphorique (Dib, 1998 : 165-166). 
Quant à la deuxième métamorphose, il m’intéresse spécialement de la rappeler ici puisqu'elle implique les deux espaces géographiques que j’évoquais au début : le désert et la mer. Les images qui s’en découlent provoquent chez le lecteur une sensation de monde inversé car elles fusionnent le haut et le bas, ce qui est sur la terre et ce qui se trouve au ciel. Peu abondantes sont les images où le ciel et la terre se confondent : « Mais ce ciel, il hypnotisait Abed : une toison de bélier que c'était ; pesante de suint et de sable aux couleurs mêmes du désert au corps à corps avec son double » (Dib, $2001: 34)$.

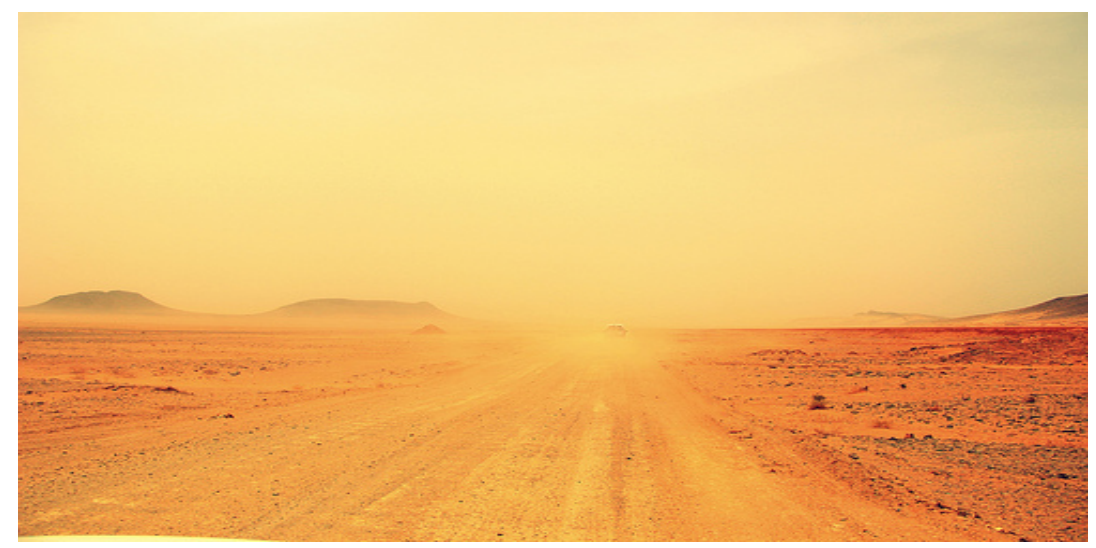

Fig. 6 Tempête de sable

photo credit: <a href="http://www.flickr.com/photos/24141546@N06/7315395230">sand storm</a > via <a href="http://photopin.com">photopin</a $><$ a href="https://creativecommons.org/licenses/by/2.0/">(license) $</ a>$

Par contre, les images où le ciel et la mer (ou d'autres grandes étendues d'eau comme les lacs) se superposent et se confondent sont très fréquentes : « Ciel, un ciel où nagent les oiseaux » (Dib, 1994 : 114). « Tellement au-dessus de la nuit, le ciel suspend son lac pâle. Je lève mes yeux pour recevoir sa fraîcheur et son pardon » (Dib, 1994 : 52). « Dans un tournant, la mer apparaît. [...] Avancer jusqu'au bord du gouffre, tentation de t’y plonger : s’y renverser pour flotter dans cette immensité, face à l'immensité renversée du ciel » (Djebar, 1987 : 28). « [...] ouvrir la fenêtre, m’imaginer que je pouvais me transformer en sirène nageant dans l'azur, quelques brasses, me voici devant sa fenêtre à lui » (Djebar, 2002 : 39).

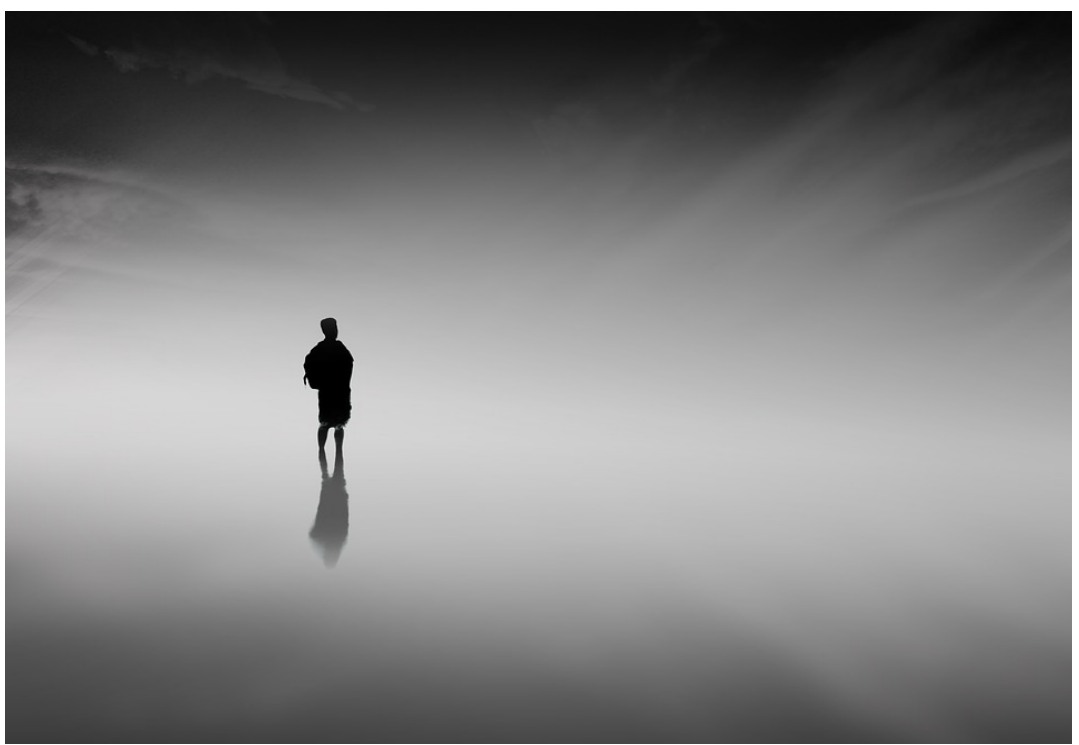

Fig. 7 Mer et ciel

Auteur: MDARIFLIMAT (Limat MD Arif)

Pixabay : CC0 Public Domain (Gratuit pour les usages commerciaux. Il n'est pas nécessaire d'en citer l'auteur) 


\section{Conclusion}

Evidemment, et je voudrais conclure sur cette ouverture, cette vision du réel où les éléments et les êtres se mélangent ou se métamorphosent les uns dans les autres grâce à la magie de l'image poétique n'est pas exclusive de ces deux auteurs ${ }^{2}$. Le lecteur la retrouve dans les romans de beaucoup d'autres auteurs maghrébins partageant un substrat islamique et parfois mystique (c'est qui est vrai pour la plupart des écrivains du Maghreb d'une façon ou d'une autre). En effet, comme je l'ai déjà avancé, dans l'art musulman (et la littérature en fait partie) tout s'unit et se répond, tout se tient. Le fait que la doctrine de l'unité ou at-tawhid constitue la base même de l'Islam, dont l'importance fondatrice pour les auteurs maghrébins n'est plus à rappeler, y est sans doute pour beaucoup.

\section{Références bibliographiques}

AraBi, A. (1989). «L’identité de l'espace esthétique dans l'art arabo-musulman ». Dans Maghreb-Machrek. Espaces et sociétés du monde arabe. Paris ( $\left.n^{\circ} 123,260-264\right)$.

BÁRcena, Halil (2008). « Rumí, música y sufismo. El samâ de los derviches giróvagos ». Choza, Jacinto et De Garay, Jesús (éds.). Dans : Danza de Oriente y danza de Occidente, Sevilla: Thémata, 2007. Publié le jeudi 22 mai 2008 dans <http://institutosufi.blogspot.com.es/2008/05/rum-y-la-msica-suf-mevlev>[Consulté le 30 juin 2016].

Belaghoueg, Zoubida (2010). «Malika Mokkedem. Du sable et de l'eau : jaillissement de la création et intinéraire d’une vie » dans Daoud. Mohamed ; Bendjelid, Faouzia et Detrez, Christine (dir.). Écriture féminine : réception, discours et représentations. Oran : Éditions CRASC.

Benali, Abdelkader (1996). Noces à la mer. Paris : Albin Michel.

BOURGET, Carine (2002). Coran et tradition islamique dans la littérature maghrébine. Paris : Karthala.

CHEBEL, Malek (1995). Dictionnaire des symboles musulmans. Rites, mystique et civilisation. Paris : Albin Michel.

CHEBEL, Malek (2002). Le Sujet en Islam. Paris : Seuil.

CHRAÏBI, Driss (1982). La Mère du Printemps. Paris : Seuil.

DiB, Mohamed (1978). La Danse du roi. Paris : Editions du Seuil.

DiB, Mohamed (1990). Neiges de marbre. Paris : Minos-La Différence.

DiB, Mohamed (1994). L'Infante maure. Paris : Albin Michel.

DiB, Mohamed (1995). La Nuit sauvage. Paris : Albin Michel.

DiB, Mohamed (1998). Si Diable veut. Paris : Albin Michel.

DiB, Mohamed (2001). Comme un bruit d'abeilles. Paris : Albin Michel.

DiB, Mohamed (2002). L'Incendie. Paris : Editions du Seuil.

DiB, Mohamed (2003a). Simorgh. Paris : Albin Michel.

DiB, Mohamed (2003b). Le Sommeil d’Ève. Paris : Minos-La Différence.

DJEBAR, Assia (1962). Les Enfants du nouveau monde. Paris : René Julliard.

DJEBAR, Assia (1983). Les Alouettes naïves. Paris. Julliard.

DJEBAR, Assia (1987). Ombre sultane. Paris : J.C. Lattès.

DJeBAR, Assia (1995). L'Amour, la fantasia. Paris : Albin Michel.

DJEBAR, Assia (1997). Les Nuits de Strasbourg. Arles : Actes Sud.

DJEBAR, Assia (1999). Ces voix qui m’assiègent...en marge de ma francophonie. Paris : Albin Michel.

DJEBAR, Assia (2002). Vaste est la prison. Paris : Albin Michel.

\footnotetext{
${ }^{2}$ Pour ne donner qu'un exemple, Carine Bourget signale que « les symboles religieux des Berbères (un poisson dans une étoile) représentent de façon métonymique l’eau et la lumière, éléments récurrents dans le Coran et dans la fiction de Chraïbi » (2002: 161).
} 
IzUTSU, Toshihiko (1997). Sufismo y Taoísmo. Ibn ‘Arabi (vol. 1). Madrid : Siruela.

LABRA Cenitagoya, Ana Isabel (sous presse). «Lumière sur lumière. Fonctions de la métaphore lumineuse dans la production narrative de Mohamed Dib et Assia Djebar ». Dans L'appréciation langagière de la Nature : le naturel, le texte et l'artifice. XXII Colloque de l'APFUE. Jaen : Sous presse.

MoKKeDEM, Malika (2001). N'zid. Paris : Éditions du Seuil.

Monleon, Ana (2007). «L’interculturalité dans l’univers poétique de Tahar Bekri » dans Quaderns de Filologia. Estudis literaris. Universitat de València (2007, vol. XII, 179-193).

MouRA, Jean-Marc (1999). Littératures francophones et théorie postcoloniale. Paris : PUF.

SOLA, Emilio (1993). Argelia entre el desierto y el mar. Madrid : Maphre.

YACINE, Kateb (1956). Nedjma. Paris : Seuil.

Zambrano, María (1977). Claros del bosque. Barcelona : Seix Barral.

Zambrano, María (1989). La tumba de Antígona. Madrid : Mondadori.

ZaouI, Amin (2014). "L’Aïd, la mer et la littérature ». Publié le 31 juillet 2014 dans < $\underline{\text { http://www.liberte- }}$ algerie.com/culture/fragments-laid-la-mer-et-la-litterature-208734/pprint/> [Consulté le 30 juin 2016]. 